\title{
Factores asociados a bajo gradiente en estenosis aórtica severa con fracción de eyección preservada
}

\author{
Julián Vega ${ }^{1}$, Luigi Gabrielli ${ }^{1}, 2$, Samuel Córdova ${ }^{1}$, Pablo Born ${ }^{3}$, Rodrigo Saavedra ${ }^{1}$, Paul McNab ${ }^{1}$. \\ 1. División de Enfermedades Cardiovasculares. Pontificia Universidad Católica de Chile. \\ 2. Advanced Center for Chronic Diseases. Escuela de Medicina. Pontificia Universidad Católica de Chile. \\ 3. Interno escuela de medicina. Pontificia Universidad Católica de Chile. \\ Sin apoyo financiero.
}

Introducción: La estenosis aórtica es frecuente en países desarrollados, cuando es severa (EAS) y sintomática se recomienda reemplazo valvular. Su diagnóstico ecocardiográfico se realiza con cualquiera de; área valvular aórtica (AVA) $<1.0 \mathrm{~cm}^{2}$, gradiente medio $(\mathrm{GM})>40 \mathrm{mmHg}$, velocidad máxima $>4 \mathrm{~m} / \mathrm{s}$. Habitualmente existe concordancia entre estos criterios, pero diversas razones generan discordancia hasta en un $40 \%$, principalmente entre área y gradiente (DAG), causando incertidumbre diagnóstica en presencia de fracción de eyección preservada del ventrículo izquierdo (FEp) (FEVI $>50 \%$ ).

Objetivos: Caracterizar pacientes con EAS en la red UC. Establecer prevalencia y factores asociados a DAG en pacientes con EAS y FEp.

Métodos: Estudio de corte transversal, incluyó todos los pacientes con $\mathrm{AVA}<1.0 \mathrm{~cm} 2$ durante 17.5 años en la red UC. Se registraron variables biodemográficas y ecocardiográficas. Los pacientes con EAS y FEp se subdividieron en 2 grupos según $\mathrm{GM}$, bajo $(\leq 40 \mathrm{mmHg})$ y alto ( $>40 \mathrm{mmHg}$ ), se utilizó t-student y Chi cuadrado.

Resultados: 1281 pacientes cumplieron criterio de AVA $<1.0 \mathrm{~cm} 2$. Edad $71.8 \pm 13$ años, mujeres $51.4 \%$, hombres el $48.6 \%$. FEVI $68.71 \pm 14.62 \%$, FEp $89,2 \%$, Características grupo GM Bajo: Edad $81.66 \pm 6.56$ años, Mujeres 56.3\%, fibrilación auricular (FA) 14.1\%. Grupo GM Alto. $68.08 \pm 13.21$ años, mujeres $47.6 \%$, FA 8\%. Presencia de DAG $42.5 \%$. Factores asociados a bajo gradiente con FEp fueron: edad avanzada (>70 años), mujer y fibrilación auricular.

Conclusiones: La EAS en nuestro medio se observa en personas mayores con FEp. La presencia de DAG es frecuente y el principal factor asociado en presencia de FEp es la FA. 


\section{Factors associated with low gradient in severe aortic valve stenosis with preserved ejection fraction}

Introduction: Aortic stenosis (AS) is the most prevalent valvular heart disease in developed countries. Symptomatic severe AS requires surgical intervention, and its echocardiographic criteria encompass: Aortic valve area $(\mathrm{AVA})<1.0 \mathrm{~cm} 2$, aortic mean gradient $(\mathrm{MG})$ $>40 \mathrm{mmHg}$, peak aortic jet velocity $>4 \mathrm{~m} / \mathrm{s}$. Usually there is agreement among these criteria, but several reasons can cause up to $40 \%$ discordant results, mainly between AVA an MG, casting doubt about severity in the setting of preserved left ventricular ejection fraction (pLVEF).

Objectives: Characterize patients with severe AS. Assess prevalence and factors related to AG discordance (AGD) in patients with pLVEF.

Methods: Cross-sectional study, that included all patients with an AVA $<1.0 \mathrm{~cm} 2$ during the last 17.5 years in UC health network. Bio-demographic and echocardiographic variables were registered. Patients with severe AS and pLVEF where allocated in 2 subgroups according to the MG as low $(\leq 40 \mathrm{mmHg})$ and high $(>40 \mathrm{mmHg}), 2$ sides t-student and chi-squared test were performed.

Results: 1281 patients fulfill criteria of $\mathrm{AVA}<1.0$ $\mathrm{cm} 2$. Age $71.8 \pm 13$ years, women $51.4 \%$, male $48.6 \%$, LVEF $68.71 \pm 14.62 \%$.pLVEF $89.2 \%$. Low MG group: Age $81.66 \pm 6.56 \mathrm{y}$, women $56.3 \%$, atrial fibrillation (AF) $14.1 \%$. High MG group: Age $68.08 \pm 13.21 \mathrm{y}$, women $47.6 \%$, AF $8 \%$. AGD prevalence was $42.5 \%$. Factors related to low MG with pLVEF were; advance age $(>70 \mathrm{y})$, women and AF.

Conclusions: Severe AS occurs mainly in advance age patients with pLVEF. AGD is frequent and the foremost related factor is $\mathrm{AF}$.

Keywords (MeSH): Aortic valve stenosis, Echocardiography Doppler, Heart Valve Diseases 


\section{Introducción:}

La estenosis aórtica (EA) es la valvulopatía más frecuente en países desarrollados, siendo severa (EAS) en el 2-4\% de la población $>75$ años ${ }^{1}$. Cuando es severa y sintomática se recomienda el reemplazo valvular quirúrgico o percutáneo según el perfil de riesgo de cada paciente ${ }^{2}$. El envejecimiento de la población asociado a nuevas posibilidades de implante valvular percutáneo (TAVI) hacen que su correcto diagnóstico tenga creciente relevancia. Para ello la ecocardiografía es el examen cardinal, pues permite obtener información anatómica y funcional de la válvula aórtica, sobre su repercusión morfo-funcional en las cavidades cardíacas, además de información asociada que impactan en su manejo como otras valvulopatías y el tamaño de la aorta torácica.

Los criterios ecocardiográficos para diagnosticar una EAS están ampliamente validados y recientemente la sociedad americana de ecocardiografía (ASE) publicó una actualización al respecto ${ }^{3}$, otorgando a la presencia de cualquiera de los siguientes tres criterios una recomendación nivel I para su diagnóstico.

1. Área valvular aórtica (AVA) $<1.0 \mathrm{~cm} 2$ (mediante la ecuación de continuidad).

2. Gradiente medio transvalvular $(\mathrm{GM})>40 \mathrm{mmHg}$.

3. Velocidad máxima transvalvular $>4 \mathrm{~m} / \mathrm{s}$.

Las decisiones clínicas se toman en base a estos criterios, que en general son concordantes. Sin embargo con creciente frecuencia observamos situaciones de discordancia entre ellos ${ }^{4}$, principalmente entre el AVA calculada y el GM, situación que puede llegar al $40 \%$ de los casos de EAS $^{5}$. Dentro de esta discordancia el escenario más representativo y desafiante son los pacientes con un AVA $<1.0 \mathrm{~cm} 2$ (AVA indexada por área de superficie corporal $<0.6 \mathrm{~cm} 2 / \mathrm{m} 2$ ) asociado a gradientes medios disminuidos ( $<40 \mathrm{mmHg}$ ), pese a tener función sistólica normal del ventrículo izquierdo (FEVI $>50 \%)^{6}$, planteando la duda de si estamos frente a una EA severa o moderada. Dicho escenario puede darse asociado a un flujo transvalvular conservado o disminuido (EAS paradojal) ${ }^{7}$ y cuando son sintomáticas quedan agrupadas en la etapa D3 de la nueva clasificación de valvulopatías de la asociación americana del corazón $(\mathrm{AHA})^{8}$. Para mayor complejidad en el último tiempo Jean Dumesnil y cols ${ }^{9}$ han propuesto una clasificación de EAS basada en patrones hemodinámicos relacionados al flujo transvavular (bajo, normal) y al gradiente medio (alto, bajo), resultando así cuatro grupos de EAS que se resumen en la figura 1. Por otra
Figura 1. Patrones hemodinámicos en EAS



Bajo flujo $\leq 35 \mathrm{ml} / \mathrm{m} 2$, Flujo normal $>35 \mathrm{ml} / \mathrm{m2}$. Bajo gradiente $<40 \mathrm{mmHg}$, Alto gradiente $\geq 40 \mathrm{mmHg}$. AG: Alto gradiente. BG: Bajo gradiente. BF: Bajo flujo. FN: Flujo normal.

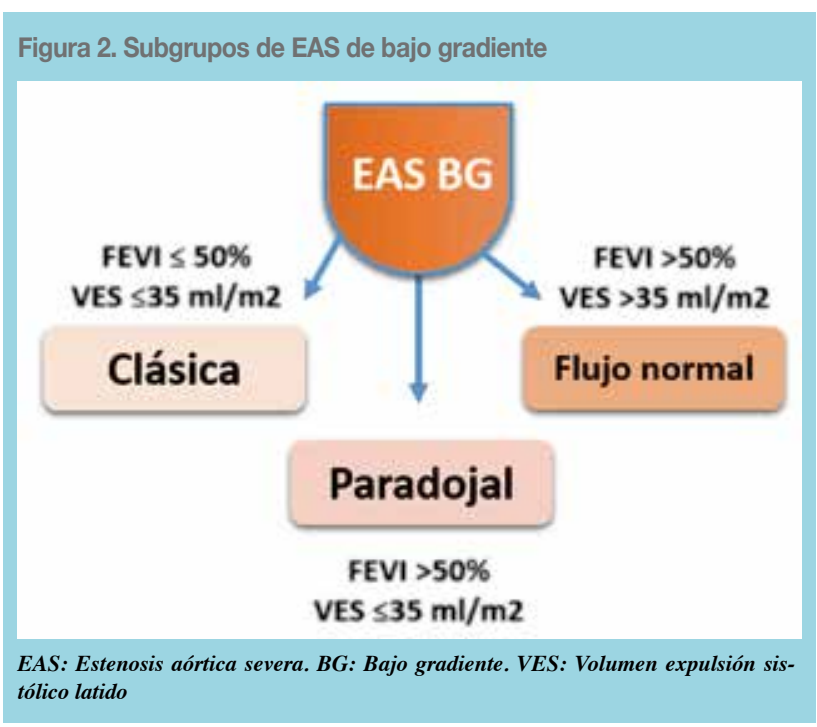

parte desde la perspectiva del bajo gradiente, existen tres posibles grupos de EAS según flujo transvalvular y FEVI que se resumen en la figura 2. Considerando lo anterior es un desafío para el ecocardiografista superar dicha discordancia, para lo cual se han propuesto varias aproximaciones que primero llaman a descartar errores técnicos, para luego integrar otras técnicas de imágenes como la ecocardiografía con Dobutamina y la tomografía computada con el puntaje de calcio de la válvula aórtica $5,10,11,12$. 
Es importante señalar que parte de esta discordancia área gradiente tiene un componente intrínseco, debido a que según la ecuación de Gorlin un GM de $40 \mathrm{mmHg}$ se corresponde con un AVA de $0.8 \mathrm{~cm} 2$ y no de $1.0 \mathrm{~cm} 2^{4}$. motivo por el cual se ha sugerido adecuar el corte de severidad a $<0.8 \mathrm{~cm}^{4},{ }^{13}$ para reconciliar la discrepancia entre área y gradiente. Sin embargo, se debe tener cautela, pues los pacientes con AVA comprendida entre $<1.0$ y $0.8 \mathrm{~cm} 2$ están expuestos a un mayor riesgo de mortalidad, independiente del gradiente ${ }^{14}$ y este grupo de pacientes se beneficia de un recambio valvular ${ }^{15}$. Agregado a lo anterior, y para obtener una mejor comprensión de esta denominada discordancia área gradiente $(\mathrm{DAG})^{16}$, es útil comprender los factores que generan un bajo gradiente transvalvular y que se esquematizan en la figura 3. Pese a la relevancia de esta situación, en nuestro medio no hay series que describan desde una perspectiva ecocardiográfica la EAS, la prevalencia de la DAG y los factores asociados a bajo gradiente en presencia de FEp. Por tanto nuestros objetivos son: realizar una caracterización ecocardiográfíca y bio-demográfica de los pacientes con EAS, establecer la prevalencia de DAG y los factores asociados a bajo gradiente con FEp.

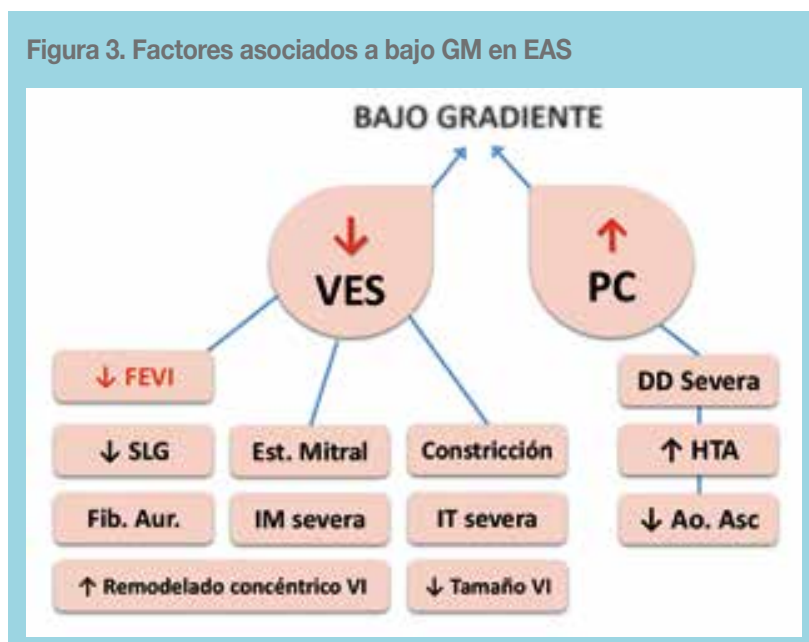

VES: Volumen expulsivo sistólico del VI. PC: Postcarga. DD: Disfunción diastólica. Ao. Asc: Aorta ascendente ( $<30 \mathrm{~mm})$. SLG: Strain longitudinal global. Fib. Aur: Fibrilación Auricular. IM: Insuficiencia mitral. IT: Insuficiencia tricuspídea.

\section{Métodos:}

Estudio de corte transversal, se incluyeron todos los pacientes con diagnóstico ecocardiográfico de EAS (definida como un AVA $<1.0 \mathrm{~cm} 2$ mediante la ecuación de continuidad) realizados en la red de salud UC duran- te los últimos 17.5 años (desde el 29.01.1998 hasta el 19.08.2015). Se registraron todos los exámenes disponibles por paciente, pero para efectos analíticos se consideró solamente el primer examen realizado. Se accedió a los estudios mediante el sistema electrónico de informe de exámenes de Cardiología (SIDEC). En una planilla ad-hoc se registraron variables biodemográficas y ecocardiográficas, las que se detallan a continuación:

Variables biodemográficas: Nombre, rut, sexo (femenino, masculino), edad (años), se definió edad avanzada $>70$ años, peso (kg), estatura (mts), área de superficie corporal (ASC) en $\mathrm{m} 2$, fecha de nacimiento.

Variables ecocardiográficas: Fecha del examen, equipo utilizado, operador.

Válvula aórtica: número de velos en tres, dos y no precisable, descripción de la válvula (engrosamiento, calcificación y restricción de la apertura de los velos, fusión de comisuras), se registró el gradiente medio y máximo en $\mathrm{mmHg}$, la integral tiempo velocidad (ITV) pre-valvular en $\mathrm{cm}$ y la insuficiencia aórtica en; ausente, leve, leve a moderada, moderada, moderada a severa y severa según las recomendaciones de la Asociación Americana de Ecocardiografía (ASE) ${ }^{17}$.

Ventrículo izquierdo: diámetro de fin de diástole (DFD), fin de sístole (DFS), grosor del septum interventricular (SIV) y de la pared posterior (PP) en fin de diástole, todos en $(\mathrm{mm})$, índice de masa en $(\mathrm{g})$, grosor parietal relativo (GPR), las variables DFD, DFS y masa del VI fueron indexadas por el ASC. El tipo de remodelado ventricular según la normativa de la $\mathrm{ASE}^{18} \mathrm{en}$ : geometría normal, remodelado concéntrico, hipertrofia concéntrica e hipertrofia excéntrica, fracción de acortamiento y la fracción de eyección (FE) por Simpson biplano. Aurícula izquierda (diámetro antero posterior en paraesternal eje largo en mm y el área en vista apical 4 cámaras $(\mathrm{A} 4 \mathrm{C})$ en $\mathrm{cm} 2$, el tamaño en fin de diástole de la raíz aórtica y de la aorta torácica ascendente en $\mathrm{mm}$ / m2. Válvula mitral; engrosamiento y calcificación de sus velos, fusión de comisuras, insuficiencia en; ausente, leve, leve a moderada, moderada, moderada a severa y severa, según la normativa ASE, grado de estenosis según el área en cm2 por planimetría bidimensional en eje corto en: ausente, leve, moderada o severa, velocidades del Doppler transmitral (onda E, onda A, tiempo de desaceleración en $\mathrm{cm} / \mathrm{s}$ ) y patrón de llenado según la normativa de la ASE en tipo 1 (Alteración de la re- 
lajación), tipo 2 (pseudo-normal), tipo 3 (restrictivo). Cavidades derechas; área de aurícula derecha en A4C en $\mathrm{cm} 2$, tamaño del ventrículo derecho a nivel basal en A4C a fin de diástole en mm y TAPSE en mm). Válvula tricúspide: insuficiencia en: ausente, leve, leve a moderada, moderada, moderada a severa y severa, estenosis en ausente, leve, moderada o severa y presión sistólica de arteria pulmonar (PSAP) en $\mathrm{mmHg}$, se consideró hipertensión pulmonar PSAP > $35 \mathrm{mmHg}$, presencia de fibrilación auricular permanente durante el examen. Todos los exámenes fueron realizados por ecocardiografistas experimentados y la cuantificación de las lesiones valvulares consideró la normativa de la ASE vigente al momento del estudio. Las variables continuas se expresaron como promedio \pm desviación estándar y las paramétricas como frecuencia absoluta. Se realizó una caracterización general de la serie, se registró el porcentaje de pacientes con DAG definido como: AVA < $1.0 \mathrm{~cm} 2$ y GM <40 mmHg. Con la finalidad de estudiar los factores asociados a bajo gradiente en presencia de FEp. De la serie total se seleccionaron los pacientes con FEVI $>50 \%$ por Simpson biplano y se dividieron en dos

\begin{tabular}{|c|c|c|c|c|}
\hline Variable & \multicolumn{4}{|c|}{ Resultado } \\
\hline DFD VI (mm) & \multicolumn{4}{|c|}{$50,89 \pm 9,32$} \\
\hline DFS VI (mm) & \multicolumn{4}{|c|}{$33 \pm 9,32$} \\
\hline SIV (mm) & \multicolumn{4}{|c|}{$13,08 \pm 2,02$} \\
\hline $\mathrm{PP}(\mathrm{mm})$ & \multicolumn{4}{|c|}{$11,86 \pm 1,92$} \\
\hline FEVI & \multicolumn{4}{|c|}{$68,71 \pm 14,62 \%$} \\
\hline $\mathrm{FEVI} \leq 50 \%$ & \multicolumn{4}{|c|}{$11 \%(n+135)$} \\
\hline FEVI $>50 \%$ & \multicolumn{4}{|c|}{$89,2 \%$ (n 1,110) } \\
\hline Tipo de remodelado del VI & Total & Fem. & Masc. & p \\
\hline Geometría normal & $5.7 \%$ & $3.06 \%$ & $8.57 \%$ & ns \\
\hline Remodelado concéntrico & $15.3 \%$ & $5.88 \%$ & $27.14 \%$ & * \\
\hline Hipertrofia concéntrica & $56.8 \%$ & $69.65 \%$ & $51.90 \%$ & * \\
\hline Hipertrofia excéntrica & $22.2 \%$ & $21.41 \%$ & $12.28 \%$ & * \\
\hline Patrón de llenado diastólico & \multicolumn{4}{|c|}{$\begin{array}{l}\text { Alteración de la relajación n } 618(48,24 \%) \\
\text { Pseudonormalizado } 270(21,08 \%) \\
\text { Restrictivo n } 82(6,40 \%)\end{array}$} \\
\hline $\begin{array}{l}\text { Fibrilación auricular } \\
\text { PSAP }\end{array}$ & \multicolumn{4}{|c|}{$\begin{array}{l}139(10.8 \%) \\
42.57 \pm 14.87 \mathrm{mmHg} .\end{array}$} \\
\hline
\end{tabular}

NOTAS: 36 pacientes tenían datos incompletos sobre las dimensiones del VI y la FEVI. 311 pacientes tenían datos incompletos sobre el patrón de llenado diastólico.

\begin{tabular}{|c|c|c|c|}
\hline & \multicolumn{3}{|c|}{ FEVI $>50 \%$} \\
\hline & BAJO & ALTO & $\mathrm{p}$ \\
\hline & $n(\%) \pm D S$ & $n(\%) \pm D S$ & \\
\hline Total (n) & $453(40,8 \%)$ & $657(59,2 \%)$ & \\
\hline Hombres n (\%) & $198(43,7 \%)$ & $344(52,4 \%)$ & * \\
\hline Mujeres n (\%) & $255(56,3 \%)$ & $313(47,6 \%)$ & * \\
\hline Edad (años) & $81,66 \pm 6,56$ & $68,08 \pm 13,21$ & ** \\
\hline Pacientes > 70 años & $428(94.5 \%)$ & $313(47 \%)$ & ** \\
\hline DTD VI indexado mm/m2 & $29,16 \pm 5,39$ & $27,79 \pm 4,83$ & ns \\
\hline DTS VI indexado mm/m2 & $18,39 \pm 4,93$ & $17,30 \pm 4,06$ & ns \\
\hline SIV VI (mm) & $12,34 \pm 1,81$ & $13,54 \pm 1,81$ & ns \\
\hline PP VI (mm) & $11,28 \pm 1,82$ & $12,20 \pm 1,79$ & ns \\
\hline FEVI \% & $74 \pm 9$ & $75 \pm 9$ & ns \\
\hline Gradiente medio & $29.65 \pm 6.77$ & $56.38 \pm 12.76$ & ** \\
\hline Masa VI indexada (g/m2) & $138,89 \pm 50,39$ & $147 \pm 46$ & \\
\hline GPR VI & $0,46 \pm 0,09$ & $0,50 \pm 0,1$ & \\
\hline Llenado restrictivo & $23(5,07 \%)$ & $31(4,7 \%)$ & ns \\
\hline IM significativa (1) & $8(1,76 \%)$ & $12(1,83 \%)$ & ns \\
\hline EM severa & $13(2,87 \%)$ & $10(1,5 \%)$ & ns \\
\hline IT significativa (1) & $15(3,3 \%)$ & $20(3 \%)$ & ns \\
\hline TAPSE (mm) & $21,62 \pm 4,16$ & $22,08 \pm 4,03$ & ns \\
\hline Disfunción del VD (2) & $10 \%$ & $8,2 \%$ & ns \\
\hline Fibrilación auricular & $64(14.1 \%)$ & $53(8 \%)$ & ** \\
\hline $\begin{array}{l}\text { (1) Se consideró insuficiencia } \\
\text { (2) Disfunción del ventrículo d } \\
\text { cia de insuficiencia tricuspídea } \\
\text { * p<0.05, ** p<0.01, ns: difere } \\
\text { DTD VI: Diámetro fin de diást } \\
\text { SIV VI: Septum interventricul } \\
\text { Grosor parietal relativo del VI }\end{array}$ & $\begin{array}{l}\text { ificativa: moderada } \\
\text { cho se consideró un } \\
\text { era. } \\
\text { no significativa. } \\
\text { del VI. DTS VI: Did } \\
\text { el VI. PP VI: Pared }\end{array}$ & $\begin{array}{l}\text { severa y severa. } \\
\text { IPSE }<17 \mathrm{~mm} \text { en } \\
\text { etro fin de sístole } \\
\text { osterior del VI. GI }\end{array}$ & \\
\hline
\end{tabular}

subgrupos, uno con GM bajo ( $<40 \mathrm{mmHg}$ ) y el otro con $\mathrm{GM}$ alto ( $\geq 40 \mathrm{mmHg}$ ). Entre estos subgrupos se evaluaron las diferencias de las variables biodemográficas y ecocardiográficas registradas. Para establecer diferencias entre dichas variables se utilizó el programa $\mathrm{R}$ y las pruebas de t-student con 2 colas y tablas de contingencias con la prueba de Chi cuadrado según correspondía, se consideró significativo un alfa $(\mathrm{p})<0.05 \mathrm{y}$ altamente significativo $<0.01$.

\section{Resultados:}

Un total de 1.281 pacientes cumplieron el criterio de una AVA $<1.0 \mathrm{~cm} 2$. Se registraron 1747 ETT (considerando los ETT de control), lo que resultó en un promedio de 
Figura 4. Resumen gráfico de los principales hallazgos ecocardiográficos

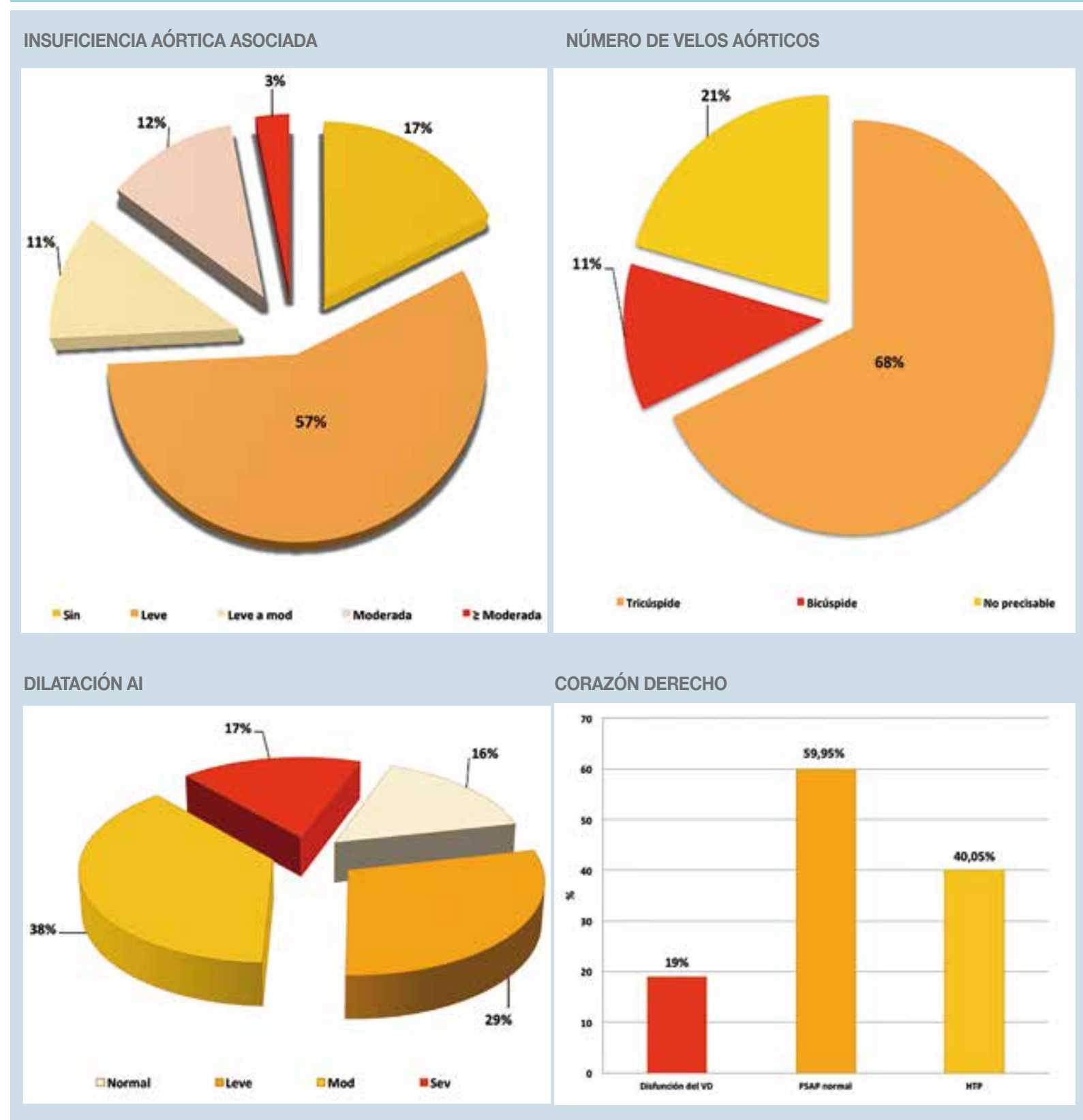

AI: Aurícula izquierda. HTP: Hipertensión pulmonar. VD: Ventrículo derecho.

1.36 estudios por paciente. Variables bio-demográficas: mujeres 51.4\% (n 659), hombres el 48.6\% (n 622), promedio de edad $71.8 \pm 13$ años. Las principales características ecocardiográficas se resumen en la tabla 1 y figura 4. La prevalencia de DAG para la serie total fue de $42.53 \%$ (n 542) y para los pacientes con FEp de $36.3 \%$ (n 403). Respecto al remodelado ventricular los hombres presentaron más remodelado concéntrico que las mujeres y estás últimas presentaron más hipertrofia, tanto concéntrica como excéntrica (Gráfico 1). En cuanto al análisis de los factores asociados a cada subgrupo según GM bajo o alto, los resultados más significativos se resumen en la tabla 2 , gráfico $1 \mathrm{y}$ gráfico 2 .

\section{Conclusiones:}

La EAS en nuestro medio se observa, principalmente, 




GN: Geometría normal. RC: Remodelado concéntrico. HC: Hipertrofia concéntrica. HE: Hipertrofia excéntrica.

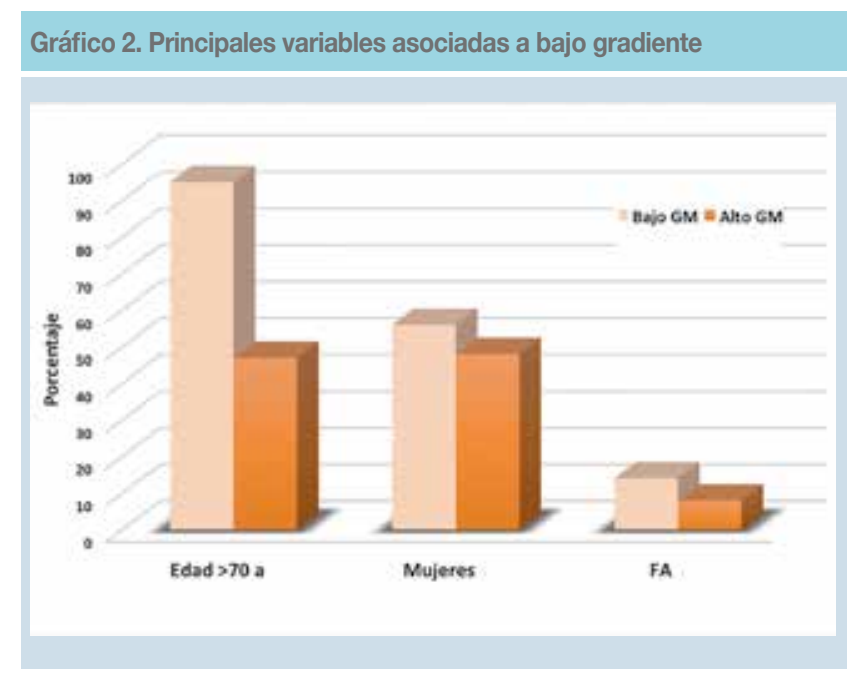

en personas mayores, similar en hombres y mujeres, con válvula de tres velos y su patrón ecocardiográfico típico es un VI remodelado con hipertrofia concéntrica, FEp, disfunción diastólica tipo 1 y sin otras valvulopatías significativas asociadas. La prevalencia de DAG en nuestra serie es similar a lo reportado por series internacionales, ocurriendo aproximadamente en 4 de cada 10 pacientes. En relación a los factores asociados a bajo gradiente con FEp, estos son; sexo femenino, edad avanzada y fibrilación auricular. Para el resto de los factores que han sido clásicamente asociados a bajo gradiente con FEp como: insuficiencia mitral o tricuspídea significativa, estenosis mitral severa, disfunción sistólica del VD y disfunción diastólica severa, en esta serie no se encontraron diferencias significativas.

\section{Discusión:}

El presente registro recopila la experiencia de los últimos 17.5 años sobre EAS, los hallazgos bio-demográficos y de morfología y función del VI están en línea con lo reportado por series internacionales ${ }^{19}$. Sin embargo, en cuanto a los factores asociados a bajo gradiente solo encontramos coincidencias en el género femenino, la edad avanzada y la presencia de fibrilación auricular, cabe destacar que este último factor tiene una fuerte e independiente asociación a bajo gradiente ${ }^{19,20}$. En cuanto a los otros factores asociados antes mencionados que en nuestra serie no se encontró diferencia significativa, cabe mencionar que tuvieron un bajo número y posiblemente baja representación en nuestro registro. Otro aspecto relevante es que no consideramos estratificar los pacientes por flujo transvalvular en bajo flujo $(\leq 35 \mathrm{ml} / \mathrm{m} 2)$ y flujo conservado ( $>35 \mathrm{ml} / \mathrm{m} 2$ ), debido a que el bajo flujo es consecuencia de una patología (insuficiencia mitral severa, disfunción ventricular izquierda, fibrilación auricular, entre otros ya mencionados) y no un problema en sí mismo. Más aún el corte arbitrario en $35 \mathrm{ml} / \mathrm{m} 2$ si bien esta validado como un predictor de mortalidad $^{21}$, es cuestionado como punto de corte de normalidad ${ }^{21}$, debido entre otros elementos a que el flujo disminuye con la edad ${ }^{22}$. Existe por consiguiente la atractiva propuesta de reemplazar dicha variable por un factor que integre el período eyectivo del ventrículo izquierdo, como es el flujo promedio transvalvular por segundo ${ }^{23}$, proponiendo un corte bajo $200 \mathrm{ml} / \mathrm{s}$ para discriminar bajo flujo ${ }^{5,24}$. No obstante, esta disyuntiva, es el gradiente medio disminuido la variable relevante que al asociarse a una AVA $<1.0 \mathrm{~cm} 2$ $(<0.6 \mathrm{~cm} 2 / \mathrm{m} 2)$ genera incertidumbre de si estamos frente a una EA severa o moderada como se hemos planteado. Finalmente, el evidenciar la magnitud del problema denominado DAG e identificar sus factores asociados, contribuye a superar inconsistencias de la cuantificación en EAS e indicar la terapia adecuada, sin privar la opción de un recambio valvular a pacientes con AVA $<1.0 \mathrm{~cm} 2$ independiente del gradiente medio asociado, circunstancia que ha sido reportada ${ }^{25}$.

\section{Limitaciones:}

Nuestra serie tiene la limitación de todo registro descriptivo, al recopilar información de exámenes ya realizados y no poder comprobar todas las mediciones. Asimismo, existían estudios con información incompleta. Otro aspecto relacionado con el gran intervalo de tiempo de nuestra serie (>17 años) es el avance en la tecnología de los equipos de ecocardiografía que permiten obtener re- 
gistros más precisos y de mejor calidad y la evolución de las guías clínicas de cuantificación ecocardiográfica con los años, sin embargo cabe señalar que los criterios de EAS se han mantenido constantes y que si bien la descripción de la EAS paradojal es relativamente nueva, la definición de EAS con bajo gradiente data de los años
$1980^{26}$. Otra arista no explorada y relevante es que no tenemos registro de la impedancia valvulo-arterial de cada examen, elemento importante a considerar cuando es la postcarga elevada la responsable del bajo gradiente transvalvular. En último lugar estamos trabajando para completar las curvas de sobrevida en los grupos descritos.

\section{Referencias}

1. CARABELlO BA, PAULUS WJ. Aortic stenosis. Lancet. 2009;373:956-66.

2. NISHIMURA RA, OTTO CM. 2017 AHA/ACC Focused Update of the 2014 AHA/ACC Guideline for the Management of Patients With Valvular Heart Disease: A Report of the American College of Cardiology/American Heart Association Task Force on Clinical Practice Guidelines. Circulation. 2017. 1-123 p.

3. Baumgartner H, Hung J, Bermejo J, Chambers JB, Edvardsen $\mathrm{T}$, Goldstein $\mathrm{S}$, et al. Recommendations on the Echocardiographic Assessment of Aortic Valve Stenosis: A Focused Update from the European Association of Cardiovascular Imaging and the American Society of Echocardiography. J Am Soc Echocardiogr. 2017;30:372-92.

4. Minners J, Allgeier M, Gohlke-Baerwolf C, Kienzle RP, Neumann FJ, Jander N. Inconsistencies of echocardiographic criteria for the grading of aortic valve stenosis. Eur Heart $\mathrm{J}$. 2008;29:1043-8.

5. Clavel M-A, Burwash IG, Pibarot P. Cardiac Imaging for Assessing Low-Gradient Severe Aortic Stenosis. JACC Cardiovasc Imaging. 2017;10:185-202.
6. Flachskampf FA. Severe aortic stenosis with low gradient and apparently preserved left ventricular systolic function - Under-recognized or overdiagnosed? Eur Heart J. 2008;29:966-8.

7. Hachicha Z, Dumesnil JG, Bogaty P, Pibarot P. Paradoxical low-flow, low-gradient severe aortic stenosis despite preserved ejection fraction is associated with higher afterload and reduced survival. Circulation. 2007;115:2856-64.

8. Nishimura R a, Otto CM, Bonow RO, Carabello B a, Erwin JP, Guyton R a, et al. 2014 AHA/ACC Guideline for the Management of Patients With Valvular Heart Disease: Executive Summary. J Am Coll Cardiol. 2014;63:2438-88.

9. Dumesnil JG, Pibarot P, Carabello B. Paradoxical low flow and/or low gradient severe aortic stenosis despite preserved left ventricular ejection fraction: Implications for diagnosis and treatment. Eur Heart J. 2010;31:281-9.

10. Tandon A, Grayburn PA. Imaging of Low-Gradient Severe Aortic Stenosis. JACC Cardiovasc Imaging. 2013;6:184-95.

11. Pibarot P, Clavel MA. Management of paradoxical low-flow, low-gradient aortic stenosis: Need for an integrated approach, including assessment of symptoms, hypertension, and stenosis severity. J Am Coll Cardiol. 2015;65:67-71. 
12. Baumgartner H, Hung J, Bermejo J, Chambers JB, Evangelista A, Griffin BP, et al. Echocardiographic Assessment of Valve Stenosis: EAE/ASE Recommendations for Clinical Practice. J Am Soc Echocardiogr. 2009;22:1-23.

13. Capoulade R, Le Ven F, Clavel M, Dumesnil J, Arsenault M, Dahou A, et al. Which Are the Best Cut-Point Value of Aortic Valve Area and Gradient to Predict Outcomes in Patients With Aortic Stenosis. Can J Cardiol. 2013;29:S158.

14. Malouf J, Le Tourneau T, Pellikka P, Sundt TM, Scott C, Schaff H V., et al. Aortic valve stenosis in community medical practice: Determinants of outcome and implications for aortic valve replacement. J Thorac Cardiovasc Surg. 2012;144:1421-7.

15. Berthelot-Richer M, Pibarot P, Capoulade R, Dumesnil JG, Dahou A, Thebault C, et al. Discordant Grading of Aortic Stenosis Severity: Echocardiographic Predictors of Survival Benefit Associated With Aortic Valve Replacement. JACC Cardiovasc Imaging. 2016;9:797-805.

16. Abbas AE, Franey LM, Goldstein J, Lester S. Aortic valve stenosis: to the gradient and beyond--the mismatch between area and gradient severity. J Interv Cardiol. 2013;26:183-94.

17. Zoghbi WA, Enriquez-Sarano M, Foster E, Grayburn PA, Kraft CD, Levine RA, et al. Recommendations for Evaluation of the Severity of Native Valvular Regurgitation with Two-dimensional and Doppler Echocardiography. J Am Soc Echocardiogr. 2003;16:777-802.

18. Lang RM, Badano LP, Mor-Avi V, Afilalo J, Armstrong A, Ernande $\mathrm{L}$, et al. Recommendations for cardiac chamber quantification by echocardiography in adults: An update from the American society of echocardiography and the European association of cardiovascular imaging. Eur Heart J Cardiovasc Imaging. 2015;16:233-71.

19. Eleid MF, Sorajja P, Michelena HI, Malouf JF, Scott CG, Pe- 1likka PA. Flow-Gradient Patterns in Severe Aortic Stenosis With Preserved Ejection Fraction: Clinical Characteristics and Predictors of Survival. Circulation. 2013;128:1781-1789.

20. Leong DP, Pizzale S, Haroun MJ, Yazdan-Ashoori P, Ladak K, Sun YY, et al. Factors Associated with Low Flow in Aortic Valve Stenosis. J Am Soc Echocardiogr. 2016; 29:158-65.

21. Eleid MF, Sorajja P, Michelena HI, Malouf JF, Scott CG, Pellikka PA. Survival by stroke volume index in patients with low-gradient normal EF severe aortic stenosis. Heart. 2015;101:23-9.

22. Strait J, Lakatta E. Aging-associated cardiovascular changes and their relationship to heart failure. Heart Fail Clin. 2012;8:143-64.

23. deFilippi CR, Willett DL, Brickner ME, Appleton CP, Yancy $\mathrm{CW}$, Eichhorn EJ, et al. Usefulness of dobutamine echocardiography in distinguishing severe from nonsevere valvular aortic stenosis in patients with depressed left ventricular function and low transvalvular gradients. Am J Cardiol. 1995;75:191-4.

24. Chahal NS, Drakopoulou M, Gonzalez-Gonzalez AM, Manivarmane R, Khattar R, Senior R. Resting Aortic Valve Area at Normal Transaortic Flow Rate Reflects True Valve Area in Suspected Low-Gradient Severe Aortic Stenosis. JACC Cardiovasc Imaging. 2015;8:1133-9.

25. Taniguchi T, Morimoto T, Shiomi H, Ando K, Kanamori N, Murata K, et al. High- Versus Low-Gradient Severe Aortic Stenosis: Demographics, Clinical Outcomes, and Effects of the Initial Aortic Valve Replacement Strategy on Long-Term Prognosis. Circ Cardiovasc Interv. 2017;10.

26. Carabello BA, Green LH, Grossman W, Cohn LH, Koster JK, Collins JJ. Hemodynamic determinants of prognosis of aortic valve replacement in critical aortic stenosis and advanced congestive heart failure. Circulation. 1980;62:42-8. 